
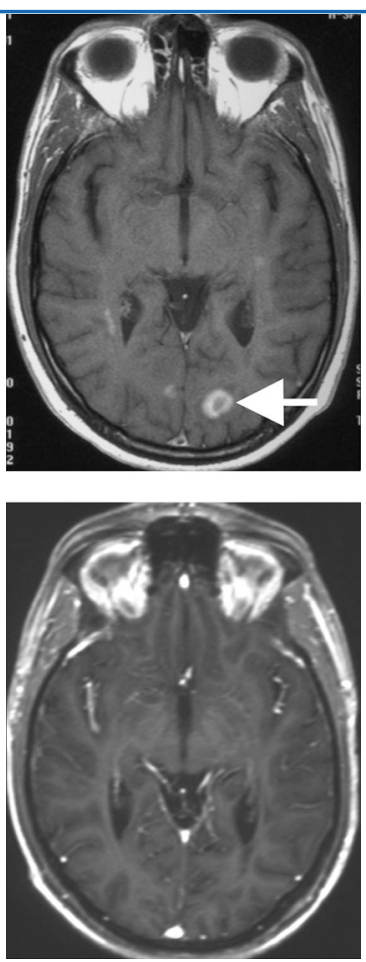

An MS lesion (arrow) was resolved two years after stem cell transplantation.

\title{
Out with the old, in with the new
}

Muraro et al., reporting on page 805 , use stem cell transplants to suppress active multiple sclerosis (MS), and then show that the treatment increased the number of naive $\mathrm{T}$ cells at the expense of the memory $\mathrm{T}$ cells that are associated with disease.

Intense immunosuppression followed by stem cell transplantation has been shown to slow or stop the formation of new brain lesions in up to $95 \%$ of patients with aggressive MS. Yet it has been controversial and rarely used, mainly because immunosuppression is risky and because nothing was known about how transplantation induced remission. One proposed theory was that transplantation might change the composition of the $\mathrm{T}$ cell pool and bias it away from autoreactivity.

Muraro and colleagues now provide the first evidence that stem cell transplantation allows patients to regenerate a new repertoire of $\mathrm{T}$ cells that are less activated and more diverse than their pretransplant repertoires. This suggests that many of the disease-causing $\mathrm{T}$ cells - most of which have a memory phenotype-were eliminated by the treatment.

However, concrete proof that autoreactive $\mathrm{T}$ cells are reduced or eliminated is difficult to obtain, partly because the specificities of the autoreactive $\mathrm{T}$ cells that invade the central nervous system are not well defined.

The difficulty in assessing the basis for treatment success is one problem. Another is the possibility that autoreactive cells will reemerge from the pool of transferred stem cells. The authors agree that these cells could eventually come back, but hope that resetting the immune system may improve the quality of life for patients with an otherwise poor prognosis.

They emphasize, however, that this intense therapy is probably only suited to a subset of MS patients who have active and aggressive disease and that its implementation will require careful patient selection. JEM

\section{Refusing to be suppressed}

On page 737, Fahlén et al. show that colitis-inducing effector T cells that cannot respond to the suppressive cytokine TGF- $\beta$ refuse to be controlled by regulatory $\mathrm{T}$ (T reg) cells.

Naturally occurring $T$ reg cells suppress the activity of effector $\mathrm{CD} 4^{+} \mathrm{T}$ cells and are essential for the prevention of autoimmune disease and chronic inflammation. TGF- $\beta$ appears to be required for T reg cell activity, but determining which cells produce TGF- $\beta$ and which cells respond to it has proven difficult.

Fahlén and colleagues now attempt to define the role of TGF- $\beta$ in a mouse model of T cell-induced colitis. In this model, colitis can be reversed when $\mathrm{CD} 4^{+} \mathrm{CD} 25^{+} \mathrm{T}$ reg cells are provided along with the disease-inducing effector cells. The authors showed that wild-type T reg cells inhibited the development of colitis induced by wild-type effector $T$ cells but failed to prevent disease caused by effector T cells bearing a signaling-defective TGF- $\beta$ receptor. This demonstrated that effector cells had to be responsive to TGF- $\beta$ to be controlled by T reg cells. The T reg cells were not required as a source of TGF- $\beta$, as T reg cells from TGF- $\beta$-deficient mice were also able to suppress. Thus, T reg cells must be prompting another cell type to produce TGF- $\beta$, or may be producing a regulatory signal that operates cooperatively with TGF- $\beta$.

But did the T reg cells also require TGF- $\beta$ signals to induce suppression, as several previous studies had shown? The authors found no differences between the T reg populations in mice with normal or signaling-defective TGF- $\beta$ receptors, and lymph node-derived T reg cells from both mice suppressed colitis.

Transfer of splenic T reg cells with mutant TGF- $\beta$ receptors, however, triggered rather than inhibited colitis. The authors suggest that this can be explained by contaminating effector cells that are also mutant for the TGF- $\beta$ receptor and, therefore, cannot be controlled by the inhibitory signal. Thus, the requirement for TGF- $\beta$ in T reg cell suppression appears to be dictated primarily by the effector cells. JEM

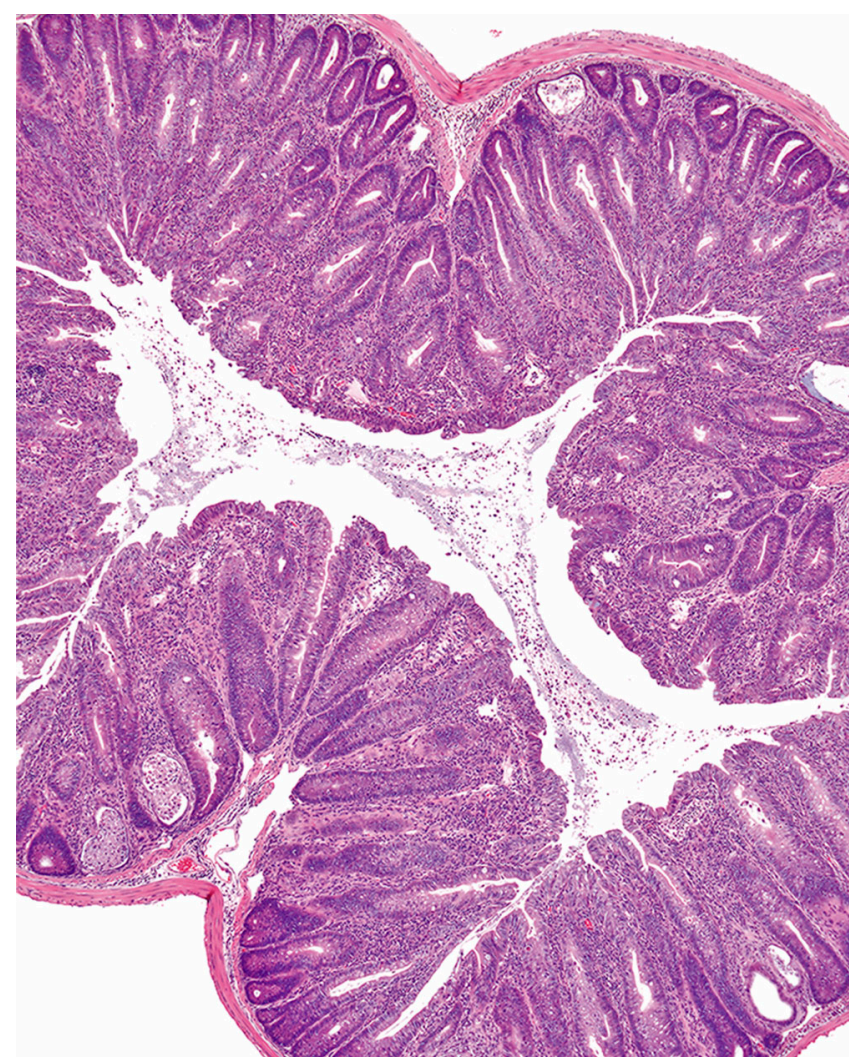

T reg cells cannot control colitis if the diseaseinducing effector $\mathrm{T}$ cells are unresponsive to TGF- $\beta$. 